\title{
Complex linear Goldstino superfield and supergravity
}

\section{Sergei M. Kuzenko}

School of Physics M013, The University of Western Australia, 35 Stirling Highway, Crawley W.A. 6009, Australia

E-mail: sergei.kuzenko@uwa.edu.au

ABSTRACT: The complex linear Goldstino superfield was proposed in arXiv:1102.3042 for the cases of global and local four-dimensional $\mathcal{N}=1$ supersymmetry. Here we make use of this superfield to construct a supergravity action which is invariant under spontaneously broken local $\mathcal{N}=1$ supersymmetry and has a positive cosmological constant for certain values of the parameters.

KeYwords: Supersymmetry Breaking, Superspaces, Supergravity Models

ArXIV EPRINT: 1508.03190 


\section{Contents}

1 Introduction 1

2 Coupling to supergravity 2

3 Super-Weyl invariant reformulation 3

4 Conclusion 4

\section{Introduction}

Four years ago, we constructed the Goldstino model [1] described by a complex linear superfield $\Sigma$ constrained by

$$
-\frac{1}{4} \bar{D}^{2} \Sigma=f, \quad f=\text { const } .
$$

Here $f$ is a parameter of mass dimension 2 which, without loss of generality, can be chosen to be real. To describe the Goldstino dynamics, $\Sigma$ was subject to the nonlinear constraints:

$$
\begin{aligned}
\Sigma^{2} & =0, \\
-\frac{1}{4} \Sigma \bar{D}^{2} D_{\alpha} \Sigma & =f D_{\alpha} \Sigma .
\end{aligned}
$$

The constraint (1.2) means that $\Sigma$ is nilpotent. The constraints (1.1), (1.2) and (1.3) imply that all component fields of $\Sigma$ are constructed in terms of a single spinor field $\bar{\rho}^{\dot{\alpha}}$. We recall that the general solution to the constraint (1.1) is

$$
\Sigma(\theta, \bar{\theta})=\mathrm{e}^{\mathrm{i} \theta \sigma^{a} \bar{\theta} \partial_{a}}\left(\phi+\theta \psi+\sqrt{2} \bar{\theta} \bar{\rho}+\theta^{2} F+\bar{\theta}^{2} f+\theta^{\alpha} \bar{\theta}^{\dot{\alpha}} U_{\alpha \dot{\alpha}}+\theta^{2} \bar{\theta} \bar{\chi}\right) .
$$

The general solution to the constraint (1.2) fixes $\phi$ and two of the auxiliary fields

$$
f \phi=\frac{1}{2} \bar{\rho}^{2}, \quad f \psi_{\alpha}=\frac{1}{\sqrt{2}} U_{\alpha \dot{\alpha}} \bar{\rho}^{\dot{\alpha}}, \quad f F=\frac{1}{\sqrt{2}} \bar{\chi} \bar{\rho}+\frac{1}{4} U^{a} U_{a} .
$$

Finally, taking into account the constraint (1.3) fixes all of the components as functions of the Goldstino $\bar{\rho}$. The explicit expressions are:

$$
\begin{aligned}
& f \phi=\frac{1}{2} \bar{\rho}^{2}, \quad \sqrt{2} f^{2} \psi_{\alpha}=-\mathrm{i} \bar{\rho}^{2}(\partial \bar{\rho})_{\alpha}, \quad f^{3} F=\bar{\rho}^{2}\left(\partial_{a} \bar{\rho} \tilde{\sigma}^{a b} \partial_{b} \bar{\rho}\right), \\
& f U_{\alpha \dot{\alpha}}=2 \mathrm{i}\left(\sigma^{a} \bar{\rho}\right)_{\alpha} \partial_{a} \bar{\rho}_{\dot{\beta}}, \quad f^{2} \bar{\chi}_{\dot{\alpha}}=\sqrt{2}\left(\left(\bar{\rho} \tilde{\sigma}^{a} \sigma^{b} \partial_{b} \bar{\rho}\right) \partial_{a} \bar{\rho}_{\dot{\beta}}-\frac{1}{2}\left(\square \bar{\rho}^{2}\right) \bar{\rho}_{\dot{\beta}}\right) .
\end{aligned}
$$


The form of the Goldstino action coincides with the free action for the complex linear superfield,

$$
S[\Sigma, \bar{\Sigma}]=-\int \mathrm{d}^{4} x \mathrm{~d}^{2} \theta \mathrm{d}^{2} \bar{\theta} \Sigma \bar{\Sigma}
$$

At the component level, this action was shown [1] to be the same as the one described by Samuel and Wess [2]. A nonlinear field redefinition relating the component form of (1.7) to the Volkov-Akulov action [3, 4] follows from the results in [5].

It was also shown in [1] that all known Goldstino superfields [2, 6-8] can be obtained as composites constructed from spinor covariant derivatives of $\Sigma$ and its conjugate (see also $[10,11]) .{ }^{1}$ This property and the universality $[3,4,12,13]$ of the Goldstino $[3,4]$ implies that any model for global supersymmetry breaking can be described in terms of $\Sigma$ and its conjugate.

Couplings of the complex linear Goldstino superfield to supersymmetric matter and $\mathcal{N}=1$ supergravity were given in [1]. The results of [1] make it possible to derive a simple construction of models for spontaneously broken $\mathcal{N}=1$ supergravity, similar to the old chiral construction of [8]. Recently, there have appeared models for spontaneously broken local supersymmetry [14, 15], which are based on the use of the chiral Goldstino superfield proposed in $[16,17]$. Here we demonstrate how the complex linear Goldstino of [1] can be used to describe spontaneously broken supergravity.

\section{Coupling to supergravity}

The supergravity generalisation ${ }^{2}$ of the constraints (1.1) and (1.3) given in [1] is as follows:

$$
\begin{aligned}
-\frac{1}{4}\left(\overline{\mathcal{D}}^{2}-4 R\right) \Sigma & =Y, \quad \overline{\mathcal{D}}_{\dot{\alpha}} Y=0, \\
-\frac{1}{4} \Sigma\left(\overline{\mathcal{D}}^{2}-4 R\right) \mathcal{D}_{\alpha} \Sigma & =Y \mathcal{D}_{\alpha} \Sigma
\end{aligned}
$$

for some covariantly chiral scalar $Y{ }^{3}$ Here $\mathcal{D}_{A}=\left(\mathcal{D}_{a}, \mathcal{D}_{\alpha}, \overline{\mathcal{D}}^{\dot{\alpha}}\right)$ denote the superspace covariant derivative corresponding to the Wess-Zumino formulation for $\mathcal{N}=1$ supergravity [19] (which at the component level is equivalent to approaches developed in [20, 21]), and $R$ is the covariantly chiral scalar component of the superspace torsion described in terms of $R, G_{\alpha \dot{\alpha}}$ and $W_{\alpha \beta \gamma}$ (see [18] for a review). Of course, the constraints (2.1a) and (2.1b) have to be accompanied by the nilpotent condition (1.2).

Here we consider the simplest case when $Y$ is a real non-zero constant,

$$
Y=f=\text { const }
$$

\footnotetext{
${ }^{1}$ The Goldstino superfields constructed in $[1,2,6,8]$ can be derived using the general relationship between linear and nonlinear realisations of supersymmetry established in [7, 9]. In particular, the spinor Goldsino superfield advocated in [2] was first constructed in [7].

${ }^{2}$ Our conventions for $\mathcal{N}=1$ supergravity mainly correspond to [33], with the only exception that the full superspace integration measure $E$ in $(2.3)$ is denoted $E^{-1}$ in [18].

${ }^{3}$ In the super-Poincaré case, modified linear constraints of the form (2.1a) were first introduced in [22].
} 
as in the rigid supersymmetric case. To describe the dynamics of the Goldstino superfield coupled to supergravity, we propose the following action:

$$
S=-\int \mathrm{d}^{4} x \mathrm{~d}^{2} \theta \mathrm{d}^{2} \bar{\theta} E\left(\frac{3}{\boldsymbol{\kappa}^{2}}+\bar{\Sigma} \Sigma\right)+\left\{\frac{\boldsymbol{\mu}}{\boldsymbol{\kappa}^{2}} \int \mathrm{d}^{4} x \mathrm{~d}^{2} \theta \mathcal{E}+\text { c.c. }\right\},
$$

where $\boldsymbol{\kappa}$ is the gravitational coupling constant and $\boldsymbol{\mu}$ a cosmological parameter. The integration measures $E$ and $\mathcal{E}$ correspond to the full superspace and its chiral subspace, respectively. The action (2.3) describes old minimal $\mathcal{N}=1$ supergravity if $\Sigma$ and $\bar{\Sigma}$ are switched off. With the Goldstino superfields $\Sigma$ and $\bar{\Sigma}$ included, the action proves to describe spontaneously broken $\mathcal{N}=1$ supergravity.

\section{Super-Weyl invariant reformulation}

To reduce the action (2.3) to components, one can follow, e.g., the component reduction procedure described in [18]. A less tedious calculation is required if one introduces a superWeyl invariant extension of (2.3) by coupling this theory to a covariantly chiral conformal compensator $\Phi, \overline{\mathcal{D}}_{\dot{\alpha}} \Phi=0$, which is assumed to be nowhere vanishing. Such an extension can be obtained following the scheme described in [23] and based on the ideas due to Kugo and Uehara [24].

The super-Weyl transformation [25] in $\mathcal{N}=1$ old minimal supergravity is

$$
\mathcal{D}_{\alpha}^{\prime}=\mathrm{e}^{\frac{1}{2} \sigma-\bar{\sigma}}\left(\mathcal{D}_{\alpha}-\left(\mathcal{D}^{\beta} \sigma\right) M_{\alpha \beta}\right), \quad \overline{\mathcal{D}}_{\dot{\alpha}}^{\prime}=\mathrm{e}^{\frac{1}{2} \bar{\sigma}-\sigma}\left(\overline{\mathcal{D}}_{\dot{\alpha}}-\left(\overline{\mathcal{D}}^{\dot{\beta}} \bar{\sigma}\right) \bar{M}_{\dot{\beta} \dot{\alpha}}\right),
$$

where $\sigma$ is an arbitrary covariantly chiral scalar parameter, $\overline{\mathcal{D}}_{\dot{\alpha}} \sigma=0$. The super-Weyl transformation of the chiral compensator $\Phi$ is

$$
\Phi^{\prime}=\mathrm{e}^{-\sigma} \Phi
$$

A super-Weyl invariant extension of the constraints (2.1) with $X$ given by (2.2) is

$$
\begin{aligned}
-\frac{1}{4}\left(\overline{\mathcal{D}}^{2}-4 R\right) \Sigma & =f \Phi^{2}, \\
-\frac{1}{4} \Sigma\left(\overline{\mathcal{D}}^{2}-4 R\right) \mathcal{D}_{\alpha}\left(\Sigma \bar{\Phi}^{-1}\right) & =f \Phi^{2} \mathcal{D}_{\alpha}\left(\Sigma \bar{\Phi}^{-1}\right),
\end{aligned}
$$

provided the super-Weyl transformation of $\Sigma$ is chosen to be

$$
\Sigma^{\prime}=\mathrm{e}^{-\bar{\sigma}} \Sigma
$$

In the super-Weyl gauge $\Phi=1$, the constraints (3.3) reduce to (2.1) ${ }^{4}$

The super-Weyl invariant extension of the action (2.3) is

$$
S=-\int \mathrm{d}^{4} x \mathrm{~d}^{2} \theta \mathrm{d}^{2} \bar{\theta} E\left(\frac{3}{\boldsymbol{\kappa}^{2}} \bar{\Phi} \Phi+\bar{\Sigma} \Sigma\right)+\left\{\frac{\boldsymbol{\mu}}{\boldsymbol{\kappa}^{2}} \int \mathrm{d}^{4} x \mathrm{~d}^{2} \theta \mathcal{E} \Phi^{3}+\text { c.c. }\right\} .
$$

\footnotetext{
${ }^{4}$ Applying a field redefinition $\Sigma \rightarrow=\Phi^{n} \Sigma$ leads to a different super-Weyl transformation law and modifies the explicit form of constraints (3.3).
} 
This action can be reduced to components using the reduction formula (5.8.50) in [18] and imposing suitable super-Weyl gauge conditions on the components of the chiral compensator $\Phi$, following the patterns described in [23] for four-dimensional $\mathcal{N}=1$ supergravitymatter systems and also in [26] for three-dimensional $\mathcal{N}=2$ supergravity-matter theories. Upon reducing the action to components and eliminating the supergravity auxiliary fields, for the cosmological constant one obtains

$$
\Lambda=f^{2}-3 \frac{|\boldsymbol{\mu}|^{2}}{\boldsymbol{\kappa}^{2}}
$$

This value agrees with the recent results in $[14,15,27],{ }^{5}$ as well as with the ancient results $[8,30]$ (see also [31]). The cosmological constant is positive for $\boldsymbol{\kappa}^{2} f^{2}>3|\boldsymbol{\mu}|^{2}$. Since the theory possesses local supersymmetry, the Goldstino can be eaten by the gravitino, in accordance with the Higgs mechanism for local supersymmetry [30, 32] (known as the super-Higgs effect). As a result, the gravitino becomes massive.

In conclusion, we recall that the constraint (1.1) is the only way to describe $\mathcal{N}=1$ anti-de Sitter supergravity using a non-minimal scalar multiplet as compensator [33]. We have shown that the same constraint can be used to describe spontaneously broken $\mathcal{N}=1$ supergravity with a positive cosmological constant.

\section{Conclusion}

Unlike the Goldstino superfields introduced in [2,6-8], the complex linear Goldstino superfield allows nontrivial matter couplings [1]. This is achieved by choosing the chiral scalar $Y$ in (2.1) to be

$$
Y=Y\left(\varphi^{i}\right)
$$

for some matter chiral superfields $\varphi^{i}$. In the presence of matter, the action (2.3) is replaced with

$$
\begin{aligned}
S= & -\int \mathrm{d}^{4} x \mathrm{~d}^{2} \theta \mathrm{d}^{2} \bar{\theta} E\left(\frac{3}{\boldsymbol{\kappa}^{2}} \mathrm{e}^{-\frac{1}{3} K(\varphi, \bar{\varphi})}+\bar{\Sigma} \Sigma\right) \\
& +\left\{\int \mathrm{d}^{4} x \mathrm{~d}^{2} \theta \mathcal{E} \Phi^{3}\left(\frac{\boldsymbol{\mu}}{\boldsymbol{\kappa}^{2}}+W(\varphi)\right)+\text { c.c. }\right\},
\end{aligned}
$$

with $K(\varphi, \bar{\varphi})$ being the Kähler potential of a Kähler manifold and $W(\varphi)$ a superpotential. Such matter couplings are analogous to the models considered in $[14,15,27]$.

If $\Sigma$ obeys only the constraint (3.3a), the supergravity-matter system (3.5) possesses a dual formulation $[1,33]$

$$
S=-\int \mathrm{d}^{4} x \mathrm{~d}^{2} \theta \mathrm{d}^{2} \bar{\theta} E\left(\frac{3}{\boldsymbol{\kappa}^{2}} \bar{\Phi} \Phi-\bar{X} X\right)+\left\{\int \mathrm{d}^{4} x \mathrm{~d}^{2} \theta \mathcal{E} \Phi^{3}\left(\frac{\boldsymbol{\mu}}{\boldsymbol{\kappa}^{2}}+f \frac{X}{\Phi}\right)+\text { c.c. }\right\},
$$

\footnotetext{
${ }^{5}$ Actually, the explicit expression (3.6) for the cosmological constant can be obtained without any calculation. The second term in (3.6) is the standard cosmological constant in pure $\mathcal{N}=1$ supergravity [28, 29], see, e.g., section 6.1.4 in [18] for a review. The first term in (3.6) follows from eq. (2.4) in [1].
} 
where $X$ is a chiral scalar superfield, $\overline{\mathcal{D}}_{\dot{\alpha}} X=0$, with the super-Weyl transformation

$$
X^{\prime}=\mathrm{e}^{-\sigma} X .
$$

It is not clear to us how to modify the duality transformation in order to take account of the nilpotent constraint (1.2).

The action (4.3) with the chiral scalar $X$ constrained by

$$
X^{2}=0
$$

describes coupling to supergravity of the Goldstino superfield introduced in $[16,17]$. This model for spontaneously broken supergravity has been of much interest recently $[14,15,27,34,35]$, in particular since it admits a nice geometric reformulation $[27,34,35]$. Here we would like to present a slightly different derivation of such a reformulation. In what follows, $X$ is assumed to obey the nilpotent constraint (4.5).

Varying (4.3) with respect to $\Phi$ gives the equation

$$
\mathbb{R}-\boldsymbol{\mu}=\frac{2}{3} f \kappa^{2} \frac{X}{\Phi}
$$

where we have the super-Weyl invariant chiral scalar

$$
\mathbb{R}=-\frac{1}{4} \Phi^{-2}\left(\overline{\mathcal{D}}^{2}-4 R\right) \bar{\Phi}
$$

which is related to the chiral scalar $\mathcal{R}$ of [34] by the rule $\mathcal{R}=\Phi \mathbb{R}$. Due to the nilpotent constraint (4.5), the equation of motion (4.6) implies that

$$
(\mathbb{R}-\boldsymbol{\mu})^{2}=0,
$$

which has the same form as the constraint put forward in [34, 35]. Making use of (4.6) once again, the action (4.3) takes the geometric form

$$
S=\left(\frac{3}{2 f \kappa^{2}}\right)^{2} \int \mathrm{d}^{4} x \mathrm{~d}^{2} \theta \mathrm{d}^{2} \bar{\theta} E \bar{\Phi} \Phi|\mathbb{R}-\boldsymbol{\mu}|^{2}-\left\{\frac{1}{2} \frac{\boldsymbol{\mu}}{\boldsymbol{\kappa}^{2}} \int \mathrm{d}^{4} x \mathrm{~d}^{2} \theta \mathcal{E} \Phi^{3}+\text { c.c. }\right\},
$$

where $\mathbb{R}$ is subject to the constraint (4.8a). This action differs in its functional form from the one proposed in $[34,35]$. The latter coincides with the minimal supergravity action

$$
S=-\frac{3}{\boldsymbol{\kappa}^{2}} \int \mathrm{d}^{4} x \mathrm{~d}^{2} \theta \mathrm{d}^{2} \bar{\theta} E \bar{\Phi} \Phi+\left\{\frac{\boldsymbol{\mu}}{\boldsymbol{\kappa}^{2}} \int \mathrm{d}^{4} x \mathrm{~d}^{2} \theta \mathcal{E} \Phi^{3}+\text { c.c. }\right\},
$$

where $\mathbb{R}$ is subject to the nilpotent constraint

$$
(\mathbb{R}-\boldsymbol{\lambda})^{2}=0, \quad \boldsymbol{\lambda}=\frac{\boldsymbol{\mu}}{\boldsymbol{\kappa}}+\frac{1}{\sqrt{3}} \boldsymbol{\kappa} f .
$$

The two descriptions should be equivalent.

An interesting problem is to understand whether our model (3.5) admits a geometric formulation similar to (4.8) or (4.9). 


\section{Acknowledgments}

The author is grateful to Simon Tyler for discussions and comments on the manuscript. Discussions with Daniel Butter and Evgeny Ivanov are also gratefully acknowledged. This work was supported by the ARC projects DP140103925.

Open Access. This article is distributed under the terms of the Creative Commons Attribution License (CC-BY 4.0), which permits any use, distribution and reproduction in any medium, provided the original author(s) and source are credited.

\section{References}

[1] S.M. Kuzenko and S.J. Tyler, Complex linear superfield as a model for Goldstino, JHEP 04 (2011) 057 [arXiv: 1102.3042] [INSPIRE].

[2] S. Samuel and J. Wess, A superfield formulation of the non-linear realization of supersymmetry and its coupling to supergravity, Nucl. Phys. B 221 (1983) 153.

[3] D.V. Volkov and V.P. Akulov, Possible universal neutrino interaction, JETP Lett. 16 (1972) 438 [INSPIRE].

[4] D.V. Volkov and V.P. Akulov, Is the neutrino a Goldstone particle?, Phys. Lett. B 46 (1973) 109 [inSPIRE].

[5] S.M. Kuzenko and S.J. Tyler, On the Goldstino actions and their symmetries, JHEP 05 (2011) 055 [arXiv: 1102.3043] [INSPIRE].

[6] M. Roček, Linearizing the Volkov-Akulov model, Phys. Rev. Lett. 41 (1978) 451 [INSPIRE].

[7] E.A. Ivanov and A.A. Kapustnikov, General relationship between linear and nonlinear realizations of supersymmetry, J. Phys. A 11 (1978) 2375 [INSPIRE].

[8] U. Lindström and M. Roček, Constrained local superfields, Phys. Rev. D 19 (1979) 2300 [INSPIRE].

[9] E.A. Ivanov and A.A. Kapustnikov, Relation between linear and nonlinear realizations of supersymmetry, Preprint JINR-E2-10765 (1977).

[10] F. Farakos, O. Hulík, P. Kočí and R. von Unge, Non-minimal scalar multiplets, supersymmetry breaking and dualities, arXiv:1507.01885 [INSPIRE].

[11] S.M. Kuzenko and S.J. Tyler, Comments on the complex linear Goldstino superfield, arXiv: 1507.04593 [INSPIRE].

[12] E.A. Ivanov and A.A. Kapustnikov, The nonlinear realization structure of models with spontaneously broken supersymmetry, J. Phys. G 8 (1982) 167 [InSPIRE].

[13] T. Uematsu and C.K. Zachos, Structure of phenomenological Lagrangians for broken supersymmetry, Nucl. Phys. B 201 (1982) 250 [INSPIRE].

[14] E.A. Bergshoeff, D.Z. Freedman, R. Kallosh and A. Van Proeyen, Pure de Sitter supergravity, arXiv:1507.08264 [INSPIRE].

[15] F. Hasegawa and Y. Yamada, Component action of nilpotent multiplet coupled to matter in 4 dimensional $\mathcal{N}=1$ supergravity, arXiv:1507.08619 [INSPIRE].

[16] R. Casalbuoni, S. De Curtis, D. Dominici, F. Feruglio and R. Gatto, Nonlinear realization of supersymmetry algebra from supersymmetric constraint, Phys. Lett. B 220 (1989) 569 [INSPIRE]. 
[17] Z. Komargodski and N. Seiberg, From linear SUSY to constrained superfields, JHEP 09 (2009) 066 [arXiv:0907.2441] [INSPIRE].

[18] I.L. Buchbinder and S.M. Kuzenko, Ideas and methods of supersymmetry and supergravity: Or a walk through superspace, IOP, Bristol U.K. (1995), revised edition (1998), pg. 656.

[19] J. Wess and B. Zumino, Superfield Lagrangian for supergravity, Phys. Lett. B 74 (1978) 51 [INSPIRE].

[20] K.S. Stelle and P.C. West, Minimal auxiliary fields for supergravity, Phys. Lett. B 74 (1978) 330 [INSPIRE].

[21] S. Ferrara and P. van Nieuwenhuizen, The auxiliary fields of supergravity, Phys. Lett. B 74 (1978) 333 [InSPIRE].

[22] B.B. Deo and S.J. Gates, Comments on nonminimal $N=1$ scalar multiplets, Nucl. Phys. B 254 (1985) 187 [inSPIRE].

[23] S.M. Kuzenko and S.A. McCarthy, On the component structure of $N=1$ supersymmetric nonlinear electrodynamics, JHEP 05 (2005) 012 [hep-th/0501172] [INSPIRE].

[24] T. Kugo and S. Uehara, Improved superconformal gauge conditions in the $N=1$ supergravity Yang-Mills matter system, Nucl. Phys. B 222 (1983) 125 [INSPIRE].

[25] P.S. Howe and R.W. Tucker, Scale invariance in superspace, Phys. Lett. B 80 (1978) 138 [INSPIRE].

[26] S.M. Kuzenko, U. Lindström, M. Roček, I. Sachs and G. Tartaglino-Mazzucchelli, Three-dimensional $\mathcal{N}=2$ supergravity theories: from superspace to components, Phys. Rev. D 89 (2014) 085028 [arXiv: 1312.4267] [INSPIRE].

[27] I. Antoniadis, E. Dudas, S. Ferrara and A. Sagnotti, The Volkov-Akulov-Starobinsky supergravity, Phys. Lett. B 733 (2014) 32 [arXiv:1403.3269] [INSPIRE].

[28] P.K. Townsend, Cosmological constant in supergravity, Phys. Rev. D 15 (1977) 2802 [INSPIRE].

[29] M. Kaku and P.K. Townsend, Poincaré supergravity as broken superconformal gravity, Phys. Lett. B 76 (1978) 54 [INSPIRE].

[30] S. Deser and B. Zumino, Broken supersymmetry and supergravity, Phys. Rev. Lett. 38 (1977) 1433 [INSPIRE].

[31] S. Cecotti, L. Girardello and M. Porrati, Constraints on partial super-Higgs, Nucl. Phys. B 268 (1986) 295 [INSPIRE].

[32] D.V. Volkov and V.A. Soroka, Higgs Effect for Goldstone Particles with Spin 1/2, JETP Lett. 18 (1973) 312 [INSPIRE].

[33] D. Butter and S.M. Kuzenko, A dual formulation of supergravity-matter theories, Nucl. Phys. B 854 (2012) 1 [arXiv:1106. 3038] [INSPIRE].

[34] E. Dudas, S. Ferrara, A. Kehagias and A. Sagnotti, Properties of nilpotent supergravity, arXiv: 1507.07842 [INSPIRE].

[35] I. Antoniadis and C. Markou, The coupling of non-linear supersymmetry to supergravity, arXiv: 1508.06767 [INSPIRE]. 